\title{
HCPCS Level I
}

National Cancer Institute

\section{Source}

National Cancer Institute. HCPCS Levell. NCI Thesaurus. Code C155825.

Codes used to report medical procedures and professional services provided by the American Medical Association as part of their Current Procedural Terminology. 